\title{
Gene-flow through space and time: dispersal, dormancy and adaptation to changing environments
}

\author{
Rafael Rubio de Casas ${ }^{1,2,5} \cdot$ Kathleen Donohue ${ }^{3}$. \\ D. Lawrence Venable ${ }^{4}$ - Pierre-Olivier Cheptou ${ }^{2}$
}

Received: 18 February 2015/ Accepted: 13 August 2015

(C) Springer International Publishing Switzerland 2015

\begin{abstract}
Dispersal through space or time (via dormancy) determines gene flow and influences demography. Because of their functional similarities, a covariation between dispersal and dormancy is expected. Dispersal and dormancy are anatomically linked in plants, because they both depend on attributes of the seed, albeit this anatomical association is rarely considered when analyzing interactions between dispersal and dormancy. In this paper, we investigate the extent to which dispersal and dormancy can be expected to correlate and how each might influence adaptation to novel environments such as those brought on by climate change. We review the theoretical and empirical literature on the subject with a focus on seed plants. We find that although a negative correlation between dispersal and dormancy has been theoretically anticipated, several models predict deviations from this expectation under scenarios of environmental heterogeneity. The empirical evidence does not support any specific covariation pattern, likely because the interaction between dispersal and dormancy is affected by multiple environmental and developmental constraints. From a climate change perspective, the effects of dispersal and dormancy on population structure are not equivalent: dormancy-mediated gene flow is intrinsically asymmetric (from the past towards the future) whereas spatial dispersal is not necessarily directional. As a result, selection on traits linked to dormancy and dispersal might differ qualitatively. In particular, gene flow through dormancy can only be adaptive if future environmental conditions are similar to those of the past, or if it contributes to novel allelic
\end{abstract}

Rafael Rubio de Casas and Kathleen Donohue have contributed equally to this article.

Rafael Rubio de Casas

rubiodecasas@ugr.es; rafa.rubiodecasas@gmail.com

1 Estación Experimental de Zonas Áridas, EEZA-CSIC, Carretera de Sacramento s/n, Almería, Spain

2 CEFE UMR 5175, CNRS, Universite de Montpellier, Universite Paul-Valery, 7 Montpellier, EPHE, 1919 route de Mende, 34293 Montpellier Cedex 05, France

3 Department of Biology, Duke University, Durham, NC 27708, USA

4 Department of Ecology and Evolutionary Biology, University of Arizona, Tucson, AZ 85721, USA

5 Departamento de Ecologia, Universidad de Granada, 18071 Granada, Spain 
combinations. We conclude that, in spite of a long tradition of research, we are unable to anticipate a universal relationship between dispersal and dormancy. More work is needed to predict the relative contributions of spatial dispersal and dormancy to gene flow and adaptation to novel environments.

Keywords Bet-hedging · Germination · INDEHISCENT · Heterocarpy · SHATTERPROOF $\cdot$ Stabilizing selection

\section{Introduction}

Dispersal, defined as the movement of an organism from the parental patch to the patch in which it breeds, is the first determinant of gene flow. Such dispersal entails the displacement of the progeny from the parental breeding site along one or several spatial dimensions (i.e., in latitude, longitude and/or altitude). However, dispersal also has a temporal dimension, as progeny experience different conditions and different population compositions from their parents, especially in annual organisms. In particular, dormancy, a form of developmental arrest, can determine the temporal distribution of progeny that are metabolically active. Just as the location of spatial dispersal can greatly influence the environmental conditions experienced by progeny, so too can the timing of dormancy breakage and emergence determine the environmental conditions experienced by progeny at later life stages (Donohue et al. 2010; Chiang et al. 2013). Both spatial and temporal dispersal therefore can influence the distribution of progeny across heterogeneous environments in ways that influence fitness and the potential for gene flow.

The spatial and temporal aspects of dispersal can be linked, especially in plants. Germination and dormancy are environment-dependent and the timing of dispersal can determine whether a seed can germinate immediately, stays in the soil until conditions are favorable for germination or is induced into dormancy (Donohue et al. 2010). Seed dormancy is conventionally defined as a state in which a seed cannot germinate even given optimal germination conditions (Finch-Savage and Leubner-Metzger 2006). This definition distinguishes dormancy from a delay in germination that is attributable to the lack of favorable germination conditions, which is also relevant from a gene-flow perspective because it can potentially isolate reproductive individuals from each other in time. However, dormancy is the main endogenous factor influencing temporal distribution of germination. Consequently, although we acknowledge the distinct roles of dormancy and germination requirements in determining germination time, in this paper we use the term "dormancy" broadly as a metric for the temporal delay in germination, i.e., as proxy for dispersal in time.

Any trait affecting dormancy will have an effect on temporal dispersal. Some of these traits are also likely to influence dispersal in space, and this anatomical and physiological linkage is expected to impose pleiotropic constraints on the evolution of the two processes. For instance, it has been established that seed dormancy is often related to seed size, such that smaller seeds tend to be more dormant (Baskin and Baskin 2014), but seed size also influences dispersal, for instance by determining the efficacy of vectors (Soons et al. 2008; Willis et al. 2014). Diverse traits such as fruit and seed morphology and chemistry, presentation of propagules on the plants, and attraction of particular dispersal vectors have the 
potential to influence both dispersal and dormancy (Cipollini and Levey 1997; Traveset 1998). However, the extent to which such structural and ontogenetic links constrain the coevolution of dispersal and dormancy and their associated traits remains to be investigated.

Dispersal and dormancy are likely to be subject to correlated selection. First, if they are controlled by the same physical structures, selection on one will impose indirect selection on the other. The net selection on each depends on the combined strength of direct selection acting on a given trait, and the strength of indirect selection, which is a function of the magnitude of the correlation among traits (Lande and Arnold 1983). Second, dispersal and dormancy may be subjected to correlational selection such that the strength and direction on dormancy varies with the dispersal phenotype, or vice versa. This may be expected because both provide the possibility of risk reduction by averaging genotype fitness over spatial or temporal environmental variation. Additionally, dispersing away from the parent in either time or space also permits escape from crowding and sib competition and reduces the probability of inbreeding (Waser et al. 2000; Ronce 2007; Vitalis et al. 2013). Because of these shared effects on the fitness and the demographic dynamics of lineages, the spatial and temporal components of dispersal experience correlated evolutionary pressures. This has led to the theoretical expectation that they may be negatively associated, such that an increase in spatial dispersal reduces the strength of selection for increased temporal dispersal and vice versa (Buoro and Carlson 2014).

Given the correlations in both their anatomical basis and in the selective influences on their evolution, how independently can spatial dispersal and dormancy evolve? Do the shared mechanisms facilitate the coordination of adaptive trait combinations of dispersal and dormancy or constrain them? Can dispersal substitute for dormancy, or vice versa? In spite of the clear commonalities between dispersal and dormancy, the question of how complementary or substitutable they are remains unanswered. Husband and Barrett (1996) already posited that treating dormancy in the same manner as dispersal might be misleading, as dormancy can contribute to population dynamics in a qualitatively different manner. Several studies have detected significant gene flow through both dispersal and dormancy (Kalisz 1986; Lavorel et al. 1994; Bomblies et al. 2010; Falahati-Anbaran et al. 2014). This is a particularly relevant subject under a scenario of climate change, when the alleles transmitted spatially might or might not be the same that are dispersing through time (McCauley 2014). However, the evolutionary consequences of simultaneous gene flow through space and time remain to be investigated in detail, as does the joint evolution of both of these mechanisms of gene flow. In particular, the influence of seed banks on the adaptation to new environments has yet to be properly quantified.

In the present paper, we investigate the multiple interactions between dispersal and dormancy, and their role in adaption to novel environments such as those brought on by climate change. Specifically, we reviewed the theoretical and empirical literature to elucidate (a) whether empirical data document positive or negative associations between dispersal and dormancy; (b) whether dispersal and dormancy are mechanistically associated, which could potentially explain some of the observed correlations. We then discuss the consequences of dispersal and dormancy on adaptive dynamics, including (c) documented consequences of the two processes on population-genetic structure, and (d) predicted consequences of dispersal and dormancy for adaptation to future climate change. Although many of our considerations can be extended to other organisms, we have focused the paper on the paradigmatic case of seed plants. 


\section{The correlation between dispersal and dormancy}

\section{The theoretical expectation of an adaptive trade-off between dispersal and dormancy}

Spatial and temporal dispersal have long since been hypothesized to be at least partially complementary and to compensate for each other (de Boer 1968). In particular, both spatial dispersal and dormancy are considered to be "bet-hedging" strategies in unpredictable heterogeneous environments. Bet hedging occurs when progeny are distributed over space or time such that some, but not all of them, experience favorable conditions; even though not all progeny experience optimal conditions, bet-hedging reduces the risk of extinction that would otherwise occur if all progeny experienced the same environment and that environment, even if rarely, happened to be of poor quality. More formally, bet hedging maximizes not the average mean fitness but the geometric mean fitness across generations. Because population growth is an inherently multiplicative process that is very sensitive to occasional extreme values, heterogeneous environments might select for bet hedging strategies (Dempster 1955).

Fig. 1 Conceptual diagram illustrating the joint evolution of dispersal and dormancy as risk reduction strategies under spatially and temporally variable environments. The cartoon represents three patches, suitable (continuous lines) or unsuitable (dashed lines) for a population (black and white circles and triangles). The environment varies also temporally across three time intervals from $\mathrm{T}_{0}$ to $\mathrm{T}_{2}$. Lightning bolts and crossed phenotypic icons represent negative selection. Dormant propagules (black) are assumed to be able to survive across one unfavorable interval, while non-dormant (white) are assumed to not disperse across time. a Dispersal is favored over dormancy when temporal variation exists within a patch, but spatial variation also exists such that, at any given time, some patches exist that are favorable (i.e., conditions differ among patches but there is at least one favorable patch at every time step). Additionally, dormancy (Do) is assumed to have higher costs than dispersal (d). The three patches are favorable at $\mathrm{T}_{0}$ but only the top one is occupied. Migrants dispersing away from this deme colonize the other two patches. At $\mathrm{T}_{1}$ the top patch is no longer favorable, although a population of dormant propagules could potentially be present. Gene-flow via dispersal is strong between the populations in the two patches in the bottom. Because dormancy has higher costs, dormant individuals are selected against. At $\mathrm{T}_{2}$ only the central patch is favorable. Dormancy disappears from the population, either because the unfavorable period has been too long (top patch) or because selection has reduced dormancy in dispersing propagules due to its costs (bottom two patches); b dormancy is favored over dispersal when all patches can be unsuitable simultaneously and when conditions are similar in space in spite of changing in time (i.e., if a patch is favorable at any given time step it is likely to be favorable at other time steps). At $\mathrm{T}_{0}$, the three patches are favorable but only the top one is occupied (same situation as in $\mathbf{a}, \mathrm{T}_{0}$ ). At $\mathrm{T}_{1}$, all the patches are unfavorable. Dormant propagules can survive in the three patches, while dispersing-only individuals cannot establish and thus disappear from the population. At $\mathrm{T}_{2}$, the top patch is favorable again. Individuals that had remained dormant in that patch can complete their life cycle, while dormant individuals in the two other patches, now unfavorable, are lost because they cannot survive any longer; c dispersal and dormancy are simultaneously selected for when conditions at any given patch at a point in time differ from those of nearby contemporary patches and from those of the same patch in the near future, i.e., when both spatial and temporal autocorrelation are negative or null. At $\mathrm{T}_{0}$, the top panel is viable and the two others are not. At $\mathrm{T}_{1}$, all patches are unfavorable (same situation as in $\mathbf{b}, \mathrm{T}_{1}$ ). At $\mathrm{T}_{2}$, the bottom patch is favorable. Dormant individuals that reached it via spatial dispersal at $\mathrm{T}_{0}$ can reproduce. The resulting population is composed of individuals that can disperse across both space and time. Conversely, when environmental conditions at a given patch are analogous to those of nearby patches and to those of the same patch in the future (i.e., when spatial and temporal autocorrelation are positive) selection should favor non-dispersing/non-dormant phenotypes (empty circles in this depiction). Note that panels $\mathbf{a}$ and $\mathbf{b}$ represent negative trade-offs between dispersal and dormancy, while c represents a situation in which a positive association between both processes is selected for. See Buoro and Carlson (2014) and references therein 
However, spatial and temporal dispersal are only partially complementary because risks in space and time are different. Dormancy may be more effective at increasing the fitness of progeny in environments with low spatial and high temporal environmental variation. In contrast, when spatial fragmentation and temporal autocorrelation are both high, spatial dispersal might increase progeny fitness more effectively than dormancy (Fig. 1a, b).

Costs of dispersal also determine the adaptive value of spatial versus temporal dispersal (Fig. 1a). Seed-bank mortality factors reduce the benefit of dormancy and allocation costs of dispersal or extreme losses of dispersing seeds reduce the benefits of spatial dispersal. In a seminal paper, Venable and Lawlor (1980) analyzed the bet-hedging attributes of spatial dispersal and dormancy and found that, when costs of dispersal exist, a tradeoff between

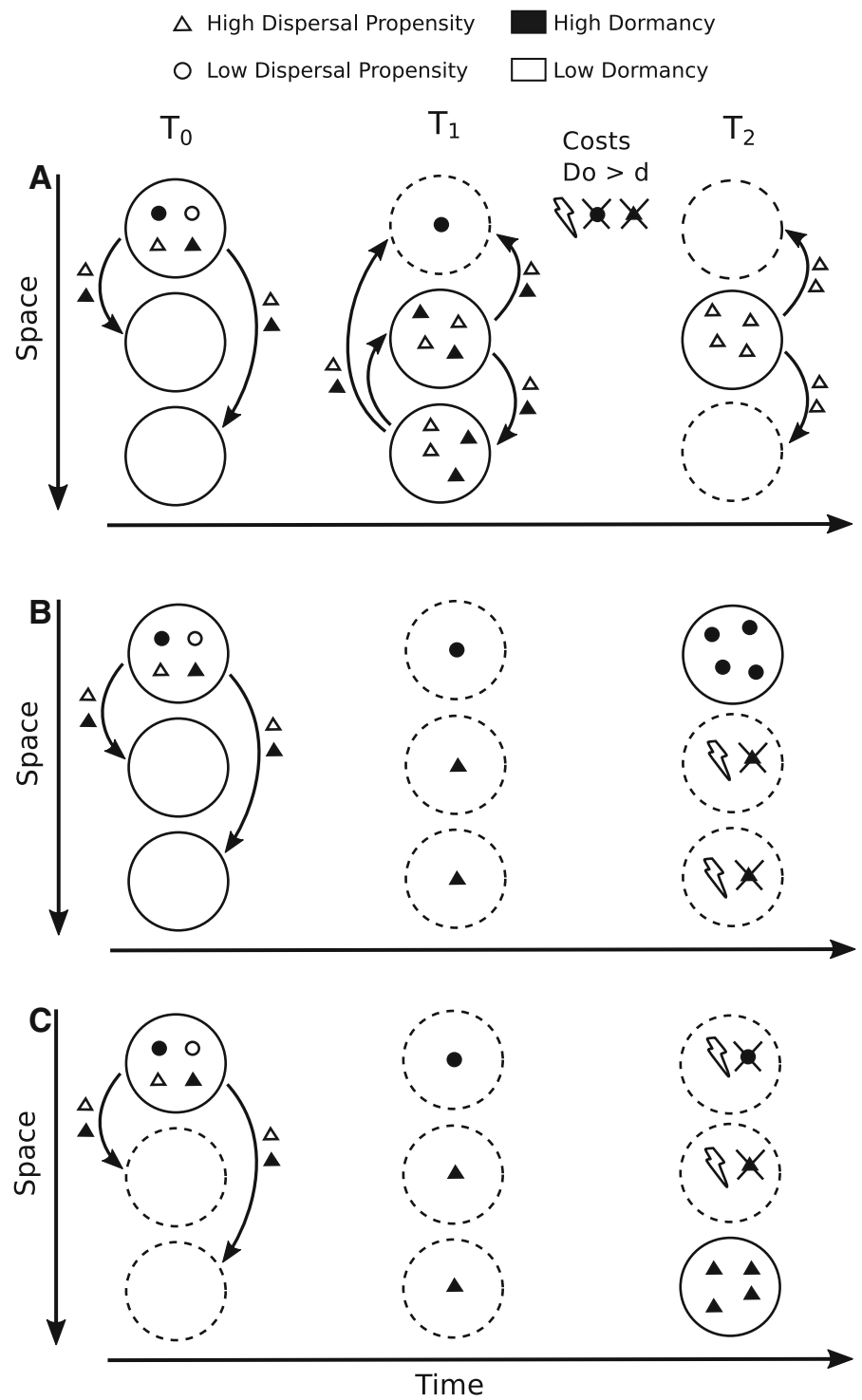


dispersal and dormancy was generally predicted. This theoretical conclusion has since been validated, at least from a theoretical perspective by other authors (Buoro and Carlson 2014). The complementary nature of dispersal and dormancy means that, all else being equal, and assuming each has some cost and that each is an effective bet-hedging strategy, having more of one reduces the need for the other. This is the basis for the expectation of a negative covariation between dispersal and dormancy.

Other theoretical studies investigating the evolution of dormancy further support the prediction that, in general, increasing spatial dispersal tends to decrease the evolutionarily stable (ES) ratio of dormant propagules (Kobayashi and Yamamura 2000; Satterthwaite 2010). Several studies analyzing the evolution of spatial dispersal also found that, in general, increasing dormancy selects for lower ES dispersal fractions (Levin et al. 1984; Cohen and Levin 1991). These models arbitrarily varied one trait and looked for correlated responses of the other. Other works have used numerical approaches to study the joint evolution of dispersal and dormancy under various ecological scenarios (Klinkhamer et al. 1987; Venable and Brown 1988; Tsuji and Yamamura 1992; Wiener and Tuljapurkar 1994; McPeek and Kalisz 1998; Olivieri 2001). These models differ in their assumptions (see Table 12.1 in Olivieri 2001, for a detailed summary) but they found that overall increased dispersal would usually select for less dormancy and vice versa (see Buoro and Carlson 2014 for a recent review).

\section{Empirically observed associations between dispersal and dormancy}

Several empirical studies have tested the trade-off between dispersal and dormancy in plants. Heterocarpic species, in which each individual simultaneously produces dispersing and non-dispersing fruits are relevant models to test this idea because fruits can often been classified a priori as dispersing or non-dispersing. For instance, in Asteraceae, the cooccurrence on individual plants of achenes bearing a pappus dispersed by wind and achenes without a pappus (a priori non-dispersing) is common (Imbert 2002) and provides a simple dichotomous dispersal trait. As such, different authors have used heterocarpic achenes to test for ecological and evolutionary correlates of differences in dispersal. Generally, it can be affirmed that non-dispersing achenes are more dormant than dispersing ones (Baskin and Baskin 2014). Heterocarpy or equivalent forms of heteromorphism in seed dispersal are also observed in other groups of plants such as the Amaranthaceae or Brassicaceae. In these groups, differences in dispersibility and dormancy between fruit morphs also seem to support a negative covariation between the two types of dispersal (see Chapter 8 in Baskin and Baskin 2014 and references therein).

However, there are also several classical empirical examples that deviate from the expectation of a negative correlation between dispersal and dormancy or even support a positive one. In the paper by Venable and Lawlor (1980), three of the 28 species studied were characterized as having both high spatial and temporal dispersal capabilities. Dispersing achenes also seem to be more dormant in Senecio jacobaea (Baker-Kratz and Maguire 1984). In other Asteraceae, there does not seem to be a clear difference in dormancy between dispersing and non-dispersing achenes (Imbert et al. 1999). Moreover, in amphicarpic plants, which produce dispersing seeds above the ground and non-dispersing subterranean seeds, the correlation appears to be positive; the subterranean seeds are generally less dormant that the aerial ones (Cheplick 1994). The phenotypes of most domesticated annual plant species, such as cereals and pulses also provide evidence that a positive correlation between dispersal and dormancy can be selected for under some circumstances. In these taxa, artificial selection has led to the loss of shattering to prevent dispersal before harvesting and in a drastic reduction of dormancy to ensure homogeneous germination of crops (Larson et al. 2014). In other words, 
loss of dispersal ability and loss of dormancy have been selected for simultaneously as part of the "domestication syndrome".

In summary, although a negative association between spatial and dormancy has frequently been found, neutral and positive associations also exist in nature. In domesticated crops, positive associations demonstrate that the correlation between spatial dispersal and dormancy can respond rapidly to selection.

\section{Predicted versus observed correlations: Should a trade-off be expected?}

The discrepancy between some empirical data and the simple theoretical prediction of a negative correlation between dispersal and dormancy might derive from the nature of models as well as from the fact that models do not incorporate constraints that are traitspecific and that might coerce associations. In most models the correlation between dispersal and dormancy is studied assuming that "all else remains equal", which is a big assumption in biology. In particular, the predicted negative correlation derives from the assumption that both dispersal and dormancy have costs and those costs are interchangeable; if one is highly effective, then expending the cost of making the other as effective is not necessarily adaptive; an increase in one trait can permit a decrease in the other. However, costs are not always comparable.

An illustrative example comes from the tradeoff between seed size and number. For a given amount of resource allocation to seeds, it is a tautologically true that there is a tradeoff between seed size and number: because seed production is always costly, if larger seeds are produced, fewer of them can be made and this has has long since been proved experimentally (Kidd and West 1918). However, there are many biological data sets showing a positive correlation between seed size and number, sometimes with accompanying text claiming that the tradeoff between seed size and number does not hold in a particular system (e.g., Schaal 1980; Koenig et al. 2009). In most such cases the tradeoff between size and number is being swamped by variation in plant size and the resources available for reproduction, i.e., by variation in the relative costs of seed production-there are large plants that make larger seeds and small plants that make fewer small seeds. In the same way, the tradeoff between dispersal and dormancy is usually true, all else being equal, but it may not always be observed because all else is not equal.

It is also important to note that the covariation of dispersal and dormancy is not always predicted to be negative when the environment changes in space and time (Fig. 1c). For example, in a model where the selective factor was escape from density, Cohen and Levin (1987) showed that dispersal and dormancy should both increase (positive correlation) if the variability of the environment increases. More recently, a model that incorporated escape from competition with both relatives and non-relatives showed that, with low extinction rates, both dispersal and dormancy are predicted to increase with decreasing population size (Vitalis et al. 2013). In general, positive correlation between dispersal and dormancy seems to be particularly likely under temporal autocorrelation of the environment (Fig. 1c; Venable and Brown 1988; Cohen and Levin 1991; Snyder 2006).

If temporal variation in environmental quality is predictable, such as seasonal changes in the environment, reduced dispersal and dormancy can evolve (Venable and Brown 1988). In contrast, when both spatial and temporal autocorrelation are negative occurs, an increase in both traits may result (Fig. 1c; Buoro and Carlsson 2014). It is challenging to establish how environmental variation is perceived by a specific organism, so it is difficult to anticipate if a given scenario of environmental variation would produce increases in both traits, decreases in both traits, or opposite responses of the two traits. 
Endogenous factors can also influence the association between dispersal and dormancy. When the effect of other traits is incorporated into models, the resulting patterns are more nuanced, even if models are kept relatively simple otherwise. In a frequently cited study, Venable and Brown (1988) considered only the risk-reducing function of dispersal and dormancy but also included seed size as a potential risk-reducing trait. They found that in the "all else being equal" scenario (same environment, when only one trait at a time varied away from the evolutionary equilibrium), negative correlations resulted in the vicinity of the evolutionary equilibrium. However, if perturbations push traits far from the combined evolutionary optimum, the direction may change and dispersal and dormancy may correlate positively. Moreover, when environments vary, all bets are off and different equilibria can be reached depending on the environmental conditions. For example, increasing the number of independently varying patches that seeds can disperse to initially selects for increased dispersal and decreased dormancy, but as the number of patches continues to increase, eventually both dispersal and dormancy decline (because there is less risk when environmental conditions are averaged across the whole system). Again, in this three-trait, density-independent system with selection resulting only from bet hedging, increasing the probability of favorable environments decreases the need for dormancy while dispersal increases initially (negative correlation) but eventually decreases (positive correlation with dormancy). Increasing dispersal distance relative to patch size favored increases of dispersal and corresponding decreases in dormancy. But as temporal autocorrelation increases, selection favors a reduction of both seed dormancy and dispersal (positive correlation).

There is empirical evidence that supports the theoretical predictions that the evolution of one trait will alter selection on the other. However, such correlational selection usually is not found to be attributable to compensation of costs of the two traits that provide similar bet-hedging benefits, but rather to specific selective consequences of exhibiting a particular dispersal or dormancy behavior. For instance, selection favoring larger, less dormant seeds might entail the selection for traits that favor long-distance dispersal, such as fleshy fruits (Bolmgren and Eriksson 2010). Conversely, a positive covariation between dispersal and dormancy might be caused by selection favoring more dormant seeds that can stay for longer periods on the maternal plant or in the soil, thus increasing the chances of primary and/or secondary dispersal (Levey and Byrne 1993; Brancalion et al. 2010). Specifically, secondary dispersal is necessarily more likely if seeds are dormant. Thus, if secondary dispersal is selected for, as seems to have been the case in several instances then dormancy might be concomitantly favored (Chambers and MacMahon 1994; Vander Wall and Longland 2004; Christianini and Oliveira 2010).

It has been predicted by several authors that certain dispersal syndromes would favor certain dormancy behaviors. In particular it has been hypothesized that fruit traits that favor dispersal by increasing retention time in animal guts should be associated to seeds with hard impermeable coats (i.e., physically dormant). Gut passage can be deleterious for seeds not protected by hard coats, whereas it might facilitate germination of physically dormant seeds through physical or chemical scarification (Gardener et al. 1993a, b; Cipollini and Levey 1997; Traveset 1998; Baldwin and Whitehead 2014; Baskin and Baskin 2014). This relationship might seem intuitive, but there is not enough data to confirm it unambiguously and studies that find no relationship between seed dormancy and gut passage abound (see Traveset 1998; Baskin and Baskin 2014 for reviews). Physical dormancy is a sophisticated anatomical mechanism to prevent imbibition of the seed which might require very specific environmental cues to open a "water gap" that might or might not be triggered by gut passage (Gama-Arachchige et al. 2013). It would be theoretically feasible to test for an association between physical dormancy and retention time using a 
controlled group of plants dispersed by the same vector. These plants should differ in their levels of seed dormancy but not in the anatomy of their water gap. Dormancy should be quantified before and after dispersal, and the potential association between predispersal dormancy level and retention time established. To our knowledge, such an experiment has never been conducted, so we cannot establish with certainty whether selection for greater retention time is associated with selection for dormancy.

Even if a negative association between spatial dispersal and dormancy is the most adaptive compromise because of their associated costs, this can be masked by variation in other traits. In general, covariation between any two traits can go undetected if a third trait is positively associated with the first two traits, and if variation in this third trait is higher than in the other two. The shared mechanistic and anatomical basis of dispersal and dormancy - with certain traits that may be positively associated with both-may weaken the predicted negative associations and even cause positive associations.

For instance, in the Asteraceae dispersing fruits are usually central achenes, whereas non-dispersing ones are peripheral. This positional difference in turn can also result in differences in dormancy even in monomorphic taxa (Baskin and Baskin 2014). In amphicarpic plants, the subterranean flowers that produce the non-dispersing seeds are usually cleistogamous (i.e., strictly self-pollinated), whereas aerial flowers are usually chasmogamous (i.e., open-pollinated) and develop in a completely different environment (Barker 2005). Both the environmental conditions of seed maturation and the degree of selfing are predicted to affect seed dormancy so they might be at least partly responsible for the differences observed in natural systems (Kobayashi and Yamamura 2000; Burghardt et al. 2015).

In addition to the traits of the propagule itself, the maternal phenotype can also influence dispersal and/or dormancy. Dispersal distance is closely associated with plant architecture, which in turn is influenced by competition (Thiede and Augspurger 1996; Wender et al. 2005), and may be subjected to selection for reasons unrelated to dispersal. The evolution of plant architecture could cause correlated evolution of dispersal. Likewise, dormancy is pleiotropically associated with flowering time (Chiang et al. 2009, 2013), so selection on flowering time could cause correlated evolution of dormancy. Consequently, the evolution of dispersal and dormancy traits might be conditioned by selection on other traits even across generations.

Undoubtedly, the fact that spatial and temporal dispersal are functions of the seed can coerce their variation. If they are both determined by the same anatomical, morphological, chemical, or phenological mechanisms, they are likely to be genetically correlated. Thus pleiotropy itself could cause positive or negative correlations between dispersal and dormancy. Our inability to predict the interdependence between dispersal and dormancy is accentuated by our limited comprehension of their developmental regulation. At this point we lack a clear understanding of the molecular mechanisms that might link them. Some of the genes that determine seed development have known pleiotropic effects on attributes that influence dispersal and dormancy, such as those that determine testa structure and characteristics in Arabidopsis thaliana. Mutants missing one or more of these genes are less dormant and maintain viability for shorter periods, which might impair their ability to disperse under natural conditions (Debeaujon et al. 2000). If that is the case, this might provide a mechanistic basis for a positive correlation: less dormancy and less dispersal in the defective mutants than in the wild type. However, to our knowledge the dispersal properties of the various testa genotypes have never been ascertained. Some of the genes that control fruit dehiscence in A. thaliana such as SPATULA and INDEHISCENT are known to influence dormancy too. Their expression seems to both facilitate dispersal by 
ensuring silique dehiscence and promote seed dormancy (Groszmann et al. 2010). Conversely, in a recent study, Chen et al. (2014) have shown that mutants with indehiscent siliques and therefore with reduced dispersal ability because of mutations in the MADSbox SHATTERPROOF genes exhibit reduced dormancy. These results might indicate that a common genetic network underlies dispersal and dormancy, but more research is needed to clarify the existence of such a network and to elucidate the genes involved and their role.

In summary, a negative correlation might be expected theoretically when considering the risk-reduction properties of dispersal and dormancy and their associated costs. This expectation of a negative correlation is observed in some natural systems, but it is by no means universally supported by theory or by empirical evidence. Deviations from the negative correlation might be caused by the pattern of environmental variation, trait covariance or phenotypic contingencies that affect trait variation. There are multiple environmental and developmental constraints that might affect the relationship between dispersal and dormancy. These constraints are poorly understood at present and therefore cannot be easily accommodated by the models, while empirical data seem to indicate that the correlation between dispersal and dormancy is flexible and can vary from positive to null to negative depending on specific selective dynamics. In spite of a long tradition of research on dispersal and dormancy, it is difficult at this point to establish a general rule for their interdependence.

\section{Dispersal, dormancy and the adaptation to novel environments}

The joint effect of dispersal and dormancy on population responses to variable environments is particularly relevant when considering climate change. Dispersal will control the capacity of species to track shifting habitat boundaries by migration, while dormancy will determine phenological tracking of the new environment. Lastly, both dispersal and dormancy will influence gene flow across demes and generations, which will in turn define the genetic variation available for adaptation to novel conditions.

Climate change has historically led to local extinctions. Palynological data have repeatedly shown that local extinctions occurred in times of past climate change, such as during the Paleocene/Eocene Thermal Maximum, the mid-Pleiocene warm interval, and interglacial periods of the Pleiostocene into the Holocene when climate change or changes in $\mathrm{CO}_{2}$ levels have been rapid, and in some cases approaching the speed of change observed today (Harrington and Jaramillo 2007; Willis et al. 2010a; Willis and MacDonald 2011). Global extinctions were prevented for many species by dispersal-enabled range shifts, which created novel patterns of species distributions and community composition. Species distributional models, moreover, have predicted extremely high extinction rates in response to future climate change, especially when dispersal is limited (Thomas et al. 2004). While these models present worst-case scenarios, they make explicit that the spatial overlap in suitable climate between the present and the future can be a critical determinant of species persistence.

Within this century, climate change has been implicated in the decline in abundance and local extinction of several flowering plant species in New England, with the extinctions occurring non-randomly with respect to phylogenetic relatedness thereby endangering entire taxonomic branches (Willis et al. 2008). The taxa most prone to local extinction were those that changed the least their flowering phenology in response to climate change, 
suggesting that phenological adjustments are important for maintaining local populations in situ (Bradshaw and Holzapfel 2008).

History tells us then that species persistence under climate change depends on environmental tracking via spatial dispersal, phenological adjustments to in situ climate, and the ability to persist in novel combinations of climatic conditions or community compositions. Each is discussed next.

\section{Environmental tracking via spatial dispersal}

One of the consequences of past climate change most frequently documented in the paleorecord is major range shifts via spatial dispersal. Indeed, in the warm periods of the Quaternary, local rather than global extinctions prevailed as species shifted their ranges altitudinally or latitudinally. Accompanying these range shifts, novel compositions of plant communities resulted, as some species migrated more efficiently than others (Botkin et al. 2007; Willis et al. 2010b, 2013).

Species distribution models have compared predicted geographic ranges under future climate scenarios assuming unlimited dispersal versus no dispersal (Peterson et al. 2002; Thomas et al. 2004). In the first case, all habitat suitable for a species is assumed to contribute to the range of that species; in the second, only suitable habitat in the future that overlaps with that in the past is assumed to be available to the species. In all cases the projected geographic ranges were severely restricted by lack of dispersal, sometimes to levels that would severely endanger the persistence of the species (Thomas et al. 2004). This result highlights the risk associated with habitat fragmentation and disrupted dispersal corridors; although migration appears to have prevented extinctions in past episodes of climate change, migration is likely to be much more limited in contemporary landscapes.

More nuanced analyses that compare different dispersal scenarios or that employ empirical estimates of dispersal parameters have been able to evaluate the role of dispersal in past extinction events and have made predictions about species distributions in the future. For example, Saltré et al. (2013) used a process-based model to predict past species distributions of forest trees, and compared scenarios with versus without dispersal, estimating dispersal from empirical data. The authors then determined whether past geographic distributions, inferred from ancient pollen records, could be explained by climate alone or whether dispersal limitation was necessary to account for the distribution of species. They were able to identify regions in the historic and present ranges that would not have been reached except for dispersal into them. Additionally, they identified the extent to which suitable locations were unoccupied because of dispersal limitation.

Spatial migration therefore appears to be one of the most important processes associated with species persistence during periods of past climate change, and either natural or assisted dispersal is likely to be important to prevent extinctions during current climate change. Importantly, spatial dispersal, although crucial for enabling species to track certain aspects of climatic conditions, has often resulted in novel conditions with respect to community composition and therefore with respect to species interactions as well as novel combinations of abiotic factors. These novel environments likely present additional challenges to which species must adapt.

\section{Phenological adjustment in response to climate}

Phenology is considered to be one of the primary factors to influence the performance of organisms during climate change (Chuine and Beaubien 2001; Walther et al. 2002; 
Parmesan and Yohe 2003; Menzel et al. 2006; Parmesan 2006; Bradshaw and Holzapfel 2008; Willis et al. 2008). Seed dormancy is an important determinant of plant phenology for two reasons. First, it determines germination time, which is itself under strong natural selection. Second, through its effect on germination time, it can influence post-germination phenology. Under climate change, the timing of seasonal changes will be altered, but it is possible that suitable environmental windows may still exist in a given location for many species. Finding that suitable temporal window for growth will be necessary to prevent exposure to stressful or fatal seasonal conditions. The temporal regulation of phenological transitions in general, and germination in particular, can be critical.

In a study conducted with annual species from the Sonoran desert, Kimball et al. (2010) showed that the timing of germination is crucial for the adaptation to the environmental conditions brought on by global change. The warming and drying of the Southwestern United States over the last decades has caused a shift in the species composition of Sonoran desert species, paradoxically favoring species that germinate and grow best in cold temperatures. Winter rains have been arriving later in the season, during periods when temperatures are lower, leading to a shift in the community composition to favor species that can germinate under cooler temperatures and that are more slow-growing, water-use efficient, and physiologically adapted to cold.

The selection on vegetative traits observed in the Sonoran plants studied by Kimball et al. (2010) also illustrates how seed dormancy, by determining germination time and consequently the seasonal environmental conditions experienced after germination, also can influence the expression of, and selection on, post-germination traits and life cycle (Donohue 2002). This idea has received further support from the recent work of Burghardt et al. (2015). In a process-based model comparing life-cycle expression of dormant versus non-dormant alleles parameterized based on Arabidopsis thaliana, they showed that the depth of seed dormancy can strongly influence the life cycle of temperate annuals under diverse climatic scenarios. Climate itself, in turn, can alter the life-history expressed by any given dormancy genotype. Their analysis showed that geographic variation in the distribution of dormancy alleles, with more dormant genotypes present at lower latitudes, interacted with climate-induced life-cycle changes, leading to the canalization of an annual life history across the geographic range. Dormancy, therefore, is a major determinant of plant responses to climate change.

\section{Gene flow in space and time: dispersal versus dormancy in response to climatic change}

As mentioned above, climate change will not only redistribute climatic conditions across the globe, but it will create novel environments and novel communities to which organisms must adapt. As such, the flow of genetic variation across time is likely to be important for adaptation to environments of the future by contributing alleles that might determine the response to selection under the new conditions.

Dormancy, in addition to being a critical component of phenological adjustment, can contribute to population persistence in time in heterogeneous environments, even if favorable periods become less frequent with global change. In fact, local extinctions are predicted to select for increased dormancy (Vitalis et al. 2013). It has already been shown that ephemeral species from arid ecosystems can persist in the seed bank for long periods 
of time and germinate following sporadic, favorable rainfall events (Facelli et al. 2005; Venable 2007).

Dormancy serves other important population-level functions, beyond hedging against catastrophic collapse. The reservoir of propagules contained in the seed-bank can have more subtle effects, such as influencing the genetic composition of populations (Kalisz 1986). In a recent experimental study of Arabidopsis thaliana Falahati-Anbaran et al. (2014) showed that gene flow via spatial dispersal introduced novel alleles to natural populations, whereas gene flow from the seed bank mitigated the variance in allele frequencies from year to year. The potential consequences of this temporal gene-flow are intriguing, particularly under changing environments.

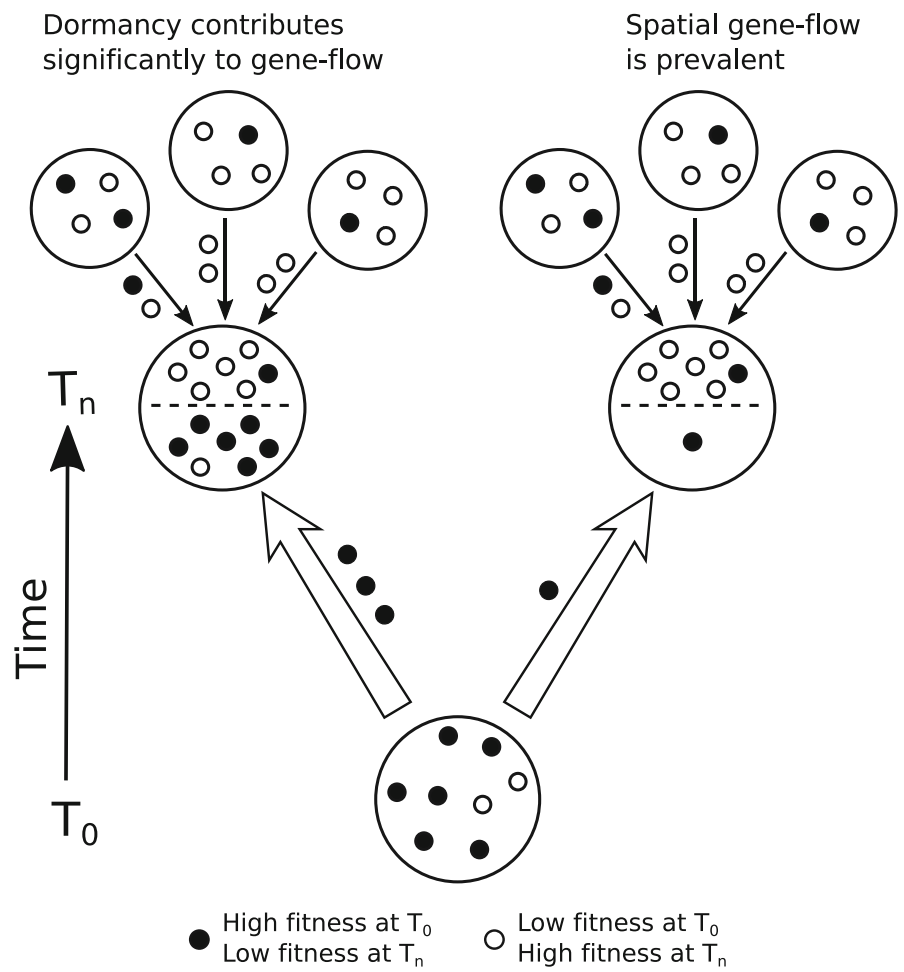

Fig. 2 Schematic illustration of the potential effect of dispersal and dormancy on adaptation to a changing environment. The figure is a cartoon representing the change in allelic frequency between time $T_{0}$ and time $\mathrm{T}_{\mathrm{n}}$ under variation of the selective regime and taking into account the contribution of both dormancy and dispersal to gene-flow. Solid circles represent genotypes favored in the ancestral patch $\left(\mathrm{T}_{0}\right)$ while empty circles are selected for at $\mathrm{T}_{\mathrm{n}}$. The dashed lines in the center of the sets at $\mathrm{T}_{\mathrm{n}}$ separate the propagules reaching the deme via dispersal (top) from those reaching it via dormancy (bottom). Selection is assumed to be spatially variable, and contemporary demes (smaller sets on the top) can contribute different alleles through dispersal. The left half of the figure depicts a scenario in which dormancy contributes significantly to geneflow. In this example, the dormant propagules that reach the patch at $T_{n}$ are not adaptive. If adaptive alleles reach the patch through dispersal, the response to selection will depend on the equilibrium between dispersal and dormancy. The right half of the cartoon represents a scenario in which the contribution of dormancy to gene-flow is significantly lower than that of spatial dispersal. If the genotypes with higher probability of reaching the new patch through dispersal are favored by selection this might foster adaptation to the new conditions 
Generally speaking, it is unclear that past genotypes are well suited to current or future climatic conditions. In a study of responses to drought, Franks et al. (2007) found evidence for rapid evolution in Brassica rapa, such that genotypes collected before a drought had lower fitness in their site of origin under present-day conditions compared to genotypes collected from those same sites after the drought. As another example, a study using ecotypes of Arabidopsis thaliana collected across a latitudinal range showed that seeds maintained from populations collected several decades ago, when planted back into the latitude from which they were collected, had lower fitness than ecotypes collected from lower latitudes (Wilczek et al. 2014). This suggests that, as climate change causes higher latitudes to have climates more similar to lower latitudes, genotypes that were historically adapted to high-latitude climates may no longer be well adapted to them.

Both examples suggest that climate change is likely to reduce the fitness of genotypes adapted to past climate conditions. This implies that long-term seed banks in situ may not be preserving the genotypes with highest fitness for present climate conditions (Fig. 2, left panel), unless temporal cycles of climate variation result in adapted dormant seeds emerging under conditions to which they are adapted again. No examples of this have been documented to our knowledge. Conversely, if the appropriate genotypes are present in other populations, spatial migration can potentially facilitate the maintenance of fitness under the new climate conditions, particularly if gene flow takes place latitudinally or altitudinally (Fig. 2, right panel).

The likelihood of dormancy and dispersal having differential effects on gene flow and adaptation depends on the correlation between them and their linkage to the traits under selection. If a positive association exists, a combination of spatial and temporal dispersal may be the best solution to adapting to climate change. If current low-latitude genotypes are adaptive in future high latitudes, then dispersal from low latitude into high latitude, combined with dormancy of those dispersed propagules, could provide alleles adapted to future climate conditions.

More data are necessary to predict the relative effect of dispersal and dormancy on population responses to changing environments. The work of Falahati-Anbaran et al. (2014) on the relative contribution of spatial and seed-bank gene flow to the genetic structure of $A$. thaliana populations might provide a useful model for the design of experiments that compare the genetic structure of the above-ground portion of populations with that of the seed bank across generations. If samples are taken before and after episodes of selection it might help us understand the relative role of dispersal and dormancy to adaptation. To increase their inference power, these experiments could be further refined by using experimental populations of known genotypes with contrasting phenotypes, similar to the experiments of Wilczek et al. (2009, 2014) or Chiang et al. (2013). These sorts of experiments could potentially shed light not only on the relative frequency of spatial versus temporal gene flow, but also on their relative contributions of adaptive alleles to natural populations in novel environments.

\section{Can gene flow from the past facilitate adaptation to future environments?}

As mentioned above, climate change will not only redistribute climatic conditions across the globe, but it will create novel environments and plant communities to which organisms must adapt. As such, the introduction of genetic variation is likely to be important for adaptation to environments of the future. The examples discussed above, in which past genotypes were less adapted to present conditions than other extant genotypes, highlight 
the importance of gene flow from other spatial locations, particularly those located at lower altitudes or latitudes, to introduce adaptive alleles.

However, gene flow even from populations adapted to past conditions can contribute to adaptive evolution in novel conditions. For multifactorial traits, especially those under stabilizing selection, the adaptive value of an allele depends on which alleles are present at other loci. Under stabilizing selection, if the phenotype of one trait is under the control of multiple loci, the optimal intermediate phenotype can be produced by a combination of alleles at different loci with enhancing ("plus" alleles) or reducing ("minus" alleles) effects on the phenotype. As such, a population that was subjected to stabilizing selection may have fixed a combination of both plus and minus alleles. Any given population, therefore, might contribute to adaptive evolution in populations with new mixtures of alleles and/or in novel environments, whether selection favors an increase or decrease in the phenotype, by providing both plus and minus alleles, even if it was optimally adapted to different environmental conditions. In contrast, directional selection would tend to fix only plus or minus alleles, depending on the direction of selection. Therefore, whether populations are likely to contribute to adaptation to present or future novel conditions depends on whether selection in the past or in other locations was stabilizing as opposed to directional.

Many phenological traits, which are so important for adaptation to climate change, have been shown to be under stabilizing selection. Germination timing in particular is likely to be subject to stabilizing selection because of antagonistic selective agents. Delayed germination can increase the probability of germinating under optimal seasonal conditions and can reduce the risk of germinating under ephemerally favorable conditions such as early spring when frost is still possible, or early in summer or autumn when drought is still likely. In contrast, early germination might increase competitive advantage and increase the length of the growing season (reviewed in Donohue et al. 2010). As a consequence, the optimal timing of germination would be some intermediate.

If selection on phenotypes that are important for climate-change adaptation, such as phenology, has been stabilizing, then genotypes (at the level of whole genomes) that do not exhibit optimally adaptive phenotypes under extant conditions may nonetheless contain alleles that could contribute to adaptation if combined with other alleles. If so, then gene flow from the past or from other populations has the potential to contribute to adaptation even when the migrant genotypes are not optimally adapted. It is interesting to speculate how the relative importance of seed banks and seed rain to adaptation to climate change might be altered, as directional changes in climate might cause more directional as opposed to stabilizing selection.

\section{Conclusions}

Dispersal and dormancy strongly influence population structure and demography. Because they share many functional commonalities, a trade-off is theoretically expected such that higher dispersibility permits lower dormancy and vice versa. However, deviations from this negative correlation are often observed in empirical data, likely because both dispersal and dormancy are determined by interactions among multiple traits and because their correlated evolution is contingent on complex selective processes.

Future research should clarify the evolutionary interdependence of dispersal and dormancy: can the traits that govern one of them change without affecting the other? Because 
dispersal and dormancy are structurally linked, it is probable that the mechanisms that regulate their evolution are constrained by pleiotropic and ontogenetic interactions. However, these putative anatomical and physiological links have not been identified explicitly in any system. Precise predictions about the correlation between dispersal and dormancy will only be possible once a good mechanistic understanding of their anatomical and physiological constraints is available.

An increase in our ability to estimate the relative contribution of dispersal and dormancy to gene flow is important to predict their role in adaptation to changing environmental conditions. Dispersal and dormancy will determine whether populations can track changing environments spatially and phenologically. Moreover, their contribution to gene flow might determine population persistence and shape the allelic combinations present in a given population and thus the chances of adaptation to the novel environment. Precise predictions of the role of temporal versus spatial gene flow to changing environments are limited by the lack of empirical data. It would be necessary to conduct studies that explicitly account for the role of dispersal and dormancy in the genetic structure of extant populations and study how these populations respond to environmental change. Furthermore, it would be interesting to test whether the relative contribution of dormancy to adaptation to future conditions depends on modes of past selection (directional or stabilizing).

Acknowledgments This work was made possible by the grant "Bet-hedging, trade-offs and the evolution of seed dispersal and dormancy" awarded to R.R.C. by the Talentia program (Junta de Andalucía/FP7) and by a 'Chercheurs d'Avenir' grant awarded to P-0. Cheptou from the Region Languedoc-Roussillon. D.L.V. was supported by the NSF Research Grant DEB 1256792.

\section{References}

Baker-Kratz AL, Maguire JD (1984) Germination and dry-matter accumulation in dimorphic achenes of tansy ragwort (Senecio jacobaea). Weed Sci 32:539-545

Baldwin JW, Whitehead SR (2014) Fruit secondary compounds mediate the retention time of seeds in the guts of Neotropical fruit bats. Oecologia 177:453-466. doi:10.1007/s00442-014-3096-2

Barker NP (2005) A review and survey of basicarpy, geocarpy, and amphicarpy in the African and Madagascan flora. Ann Mo Bot Gard 92:445-462

Baskin CC, Baskin JM (2014) Seeds ecology, biogeography and evolution of dormancy and germination. Elsevier, Amsterdam

Boer PJD (1968) Spreading of risk and stabilization of animal numbers. Acta Biotheor 18:165-194. doi:10. 1007/BF01556726

Bolmgren K, Eriksson O (2010) Seed mass and the evolution of fleshy fruits in angiosperms. Oikos 119:707-718. doi:10.1111/j.1600-0706.2009.17944.x

Bomblies K, Yant L, Laitinen RA et al (2010) Local-scale patterns of genetic variability, outcrossing, and spatial structure in natural stands of Arabidopsis thaliana. PLoS Genet. doi:10.1371/journal.pgen. 1000890

Botkin DB, Saxe H, Araújo MB et al (2007) Forecasting the effects of global warming on biodiversity. Bioscience 57:227-236. doi:10.1641/B570306

Bradshaw WE, Holzapfel CM (2008) Genetic response to rapid climate change: it's seasonal timing that matters. Mol Ecol 17:157-166. doi:10.1111/j.1365-294X.2007.03509.x

Brancalion PHS, Novembre ADLC, Rodrigues RR, Marcos Filho J (2010) Dormancy as exaptation to protect mimetic seeds against deterioration before dispersal. Ann Bot 105:991-998. doi:10.1093/aob/ mcq068

Buoro M, Carlson SM (2014) Life-history syndromes: integrating dispersal through space and time. Ecol Lett 17:756-767. doi:10.1111/ele.12275

Burghardt LT, Metcalf CJE, Wilczek AM et al (2015) Modeling the influence of genetic and environmental variation on the expression of plant life ycles across landscapes. Am Nat. doi:10.1086/679439 
Chambers JC, MacMahon JA (1994) A day in the life of a seed: movements and fates of seeds and their implications for natural and managed systems. Trends Ecol Evol 25:263-292

Chen M, MacGregor DR, Dave A et al (2014) Maternal temperature history activates Flowering Locus T in fruits to control progeny dormancy according to time of year. Proc Natl Acad Sci USA 111:18787-18792. doi:10.1073/pnas.1412274111

Cheplick GP (1994) Life history evolution in amphicarpic plants. Plant Species Biol 9:119-131. doi:10. 1111/j.1442-1984.1994.tb00092.x

Chiang GCK, Barua D, Kramer EM et al (2009) Major flowering time gene, FLOWERING LOCUS C, regulates seed germination in Arabidopsis thaliana. Proc Natl Acad Sci USA 106:11661-11666. doi:10.1073/pnas.0901367106

Chiang GCK, Barua D, Dittmar E et al (2013) Pleiotropy in the wild: the dormancy gene DOG1 exerts cascading control on life cycles. Evolution 67:883-893. doi:10.1111/j.1558-5646.2012.01828.x

Christianini AV, Oliveira PS (2010) Birds and ants provide complementary seed dispersal in a neotropical savanna. J Ecol 98:573-582. doi:10.1111/j.1365-2745.2010.01653.x

Chuine I, Beaubien EG (2001) Phenology is a major determinant of tree species range. Ecol Lett 4:500-510. doi:10.1046/j.1461-0248.2001.00261.x

Cipollini ML, Levey DJ (1997) Secondary metabolites of fleshy vertebrate-dispersed fruits: adaptive hypotheses and implications for seed dispersal. Am Nat 150:346-372. doi:10.1086/286069

Cohen D, Levin SA (1987) The interaction between dispersal and dormancy strategies in varying and heterogeneous environments. In: Teramoto E, Yumaguti M (eds) Mathematical topics in population biology, morphogenesis and neurosciences. Springer, Berlin, pp 110-122

Cohen D, Levin SA (1991) Dispersal in patchy environments: the effects of temporal and spatial structure. Theor Popul Biol 39:63-99. doi:10.1016/0040-5809(91)90041-D

Debeaujon I, Léon-Kloosterziel KM, Koornneef M (2000) Influence of the testa on seed dormancy, germination, and longevity in Arabidopsis. Plant Physiol 122:403-414. doi:10.1104/pp.122.2.403

Dempster ER (1955) Maintenance of genetic heterogeneity. Cold Spring Harb Symp Quant Biol 20:25-31 (discussion, 31-32)

Donohue K (2002) Germination timing influences natural selection on life-history characters in Arabidopsis thaliana. Ecology 83:1006-1016

Donohue K, de Casas RR, Burghardt L et al (2010) Germination, post-germination adaptation, and species ecological ranges. Annu Rev Ecol Evol Syst 41:293-319. doi:10.1146/annurev-ecolsys-102209144715

Facelli JM, Chesson P, Barnes N (2005) Differences in seed biology of annual plants in arid lands: a key ingredient of the storage effect. Ecology 86:2998-3006. doi:10.1890/05-0304

Falahati-Anbaran M, Lundemo S, Stenøien HK (2014) Seed dispersal in time can counteract the effect of gene flow between natural populations of Arabidopsis thaliana. New Phytol 202:1043-1054. doi:10. $1111 /$ nph. 12702

Finch-Savage W, Leubner-Metzger G (2006) Seed dormancy and the control of germination. New Phytol 171:501-523

Franks SJ, Sim S, Weis AE (2007) Rapid evolution of flowering time by an annual plant in response to a climate fluctuation. Proc Natl Acad Sci 104:1278-1282. doi:10.1073/pnas.0608379104

Gama-Arachchige NS, Baskin JM, Geneve RL, Baskin CC (2013) Identification and characterization of ten new water gaps in seeds and fruits with physical dormancy and classification of water-gap complexes. Ann Bot 112:69-84. doi:10.1093/aob/mct094

Gardener CJ, McIvor JG, Jansen A (1993a) Passage of legume and grass seeds through the digestive tract of cattle and their survival in faeces. J Appl Ecol 30:63-74. doi:10.2307/2404271

Gardener CJ, McIvor JG, Jansen A (1993b) Survival of seeds of tropical grassland species subjected to bovine digestion. J Appl Ecol 30:75-85. doi:10.2307/2404272

Groszmann M, Bylstra Y, Lampugnani ER, Smyth DR (2010) Regulation of tissue-specific expression of SPATULA, a bHLH gene involved in carpel development, seedling germination, and lateral organ growth in Arabidopsis. J Exp Bot 61:1495-1508. doi:10.1093/jxb/erq015

Harrington GJ, Jaramillo CA (2007) Paratropical floral extinction in the Late Palaeocene-Early Eocene. J Geol Soc 164:323-332. doi:10.1144/0016-76492006-027

Husband BC, Barrett SCH (1996) A metapopulation perspective in plant population biology. J Ecol 84:461-469. doi: $10.2307 / 2261207$

Imbert E (2002) Ecological consequences and ontogeny of seed heteromorphism. Perspect Plant Ecol Evol Syst 5:13-36

Imbert E, Escarré J, Lepart J (1999) Local adaptation and non-genetic maternal effects among three populations of Crepis sancta (Asteraceae). Ecoscience 6:223-229 
Kalisz S (1986) Variable selection on the timing of germination in Collinsia verna (Scrophulariacea). Evolution 40:479-491

Kidd F, West C (1918) Physiological pre-determination: the influence of the physiological condition of the seed upon the course of subsequent growth and upon the yield II. Review of literature. Chapter I. Ann Appl Biol 5:112-142. doi:10.1111/j.1744-7348.1918.tb05286.x

Kimball S, Angert AL, Huxman TE, Venable DL (2010) Contemporary climate change in the Sonoran Desert favors cold-adapted species. Glob Change Biol 16:1555-1565. doi:10.1111/j.1365-2486.2009. 02106.x

Klinkhamer PGL, de Jong TJ, Metz JAJ, Val J (1987) Life history tactics of annual organisms: the joint effects of dispersal and delayed germination. Theor Popul Biol 32:127-156. doi:10.1016/00405809(87)90044-X

Kobayashi Y, Yamamura N (2000) Evolution of seed dormancy due to sib competition: effect of dispersal and inbreeding. J Theor Biol 202:11-24

Koenig WD, Knops JMH, Carmen WJ, Sage RD (2009) No trade-off between seed size and number in the Valley Oak Quercus lobata. Am Nat 173:682-688. doi:10.1086/597605

Lande R, Arnold SJ (1983) The measurement of selection on correlated characters. Evolution 37:1210-1226

Larson G, Piperno DR, Allaby RG et al (2014) Current perspectives and the future of domestication studies. Proc Natl Acad Sci 111:6139-6146. doi:10.1073/pnas.1323964111

Lavorel S, Lepart J, Debussche M et al (1994) Small scale disturbances and the maintenance of species diversity in Mediterranean old fields. Oikos 70:455-473

Levey DJ, Byrne MM (1993) Complex ant-plant interactions: rain-forest ants as secondary dispersers and post-dispersal seed predators. Ecology 74:1802-1812. doi:10.2307/1939938

Levin SA, Cohen D, Hastings A (1984) Dispersal strategies in patchy environments. Theor Popul Biol 26:165-191. doi:10.1016/0040-5809(84)90028-5

McCauley DE (2014) What is the influence of the seed bank on the persistence and genetic structure of plant populations that experience a high level of disturbance? New Phytol 202:734-735. doi:10.1111/nph. 12732

McPeek MA, Kalisz S (1998) On the joint evolution of dispersal and dormancy in metapopulations. Ergeb Limnol 52:33-51

Menzel A, Sparks TH, Estrella N et al (2006) European phenological response to climate change matches the warming pattern. Glob Change Biol 12:1969-1976. doi:10.1111/j.1365-2486.2006.01193.x

Olivieri I (2001) The evolution of seed heteromorphism in a metapopulation: interactions between dispersal and dormancy. pp 245-266

Parmesan C (2006) Ecological and evolutionary responses to recent climate change. Annu Rev Ecol Evol Syst 37:637-669. doi:10.1146/annurev.ecolsys.37.091305.110100

Parmesan C, Yohe G (2003) A globally coherent fingerprint of climate change impacts across natural systems. Nature 421:37-42. doi:10.1038/nature01286

Peterson AT, Ortega-Huerta MA, Bartley J et al (2002) Future projections for Mexican faunas under global climate change scenarios. Nature 416:626-629. doi:10.1038/416626a

Ronce O (2007) How does it feel to be like a rolling stone? Ten questions about dispersal evolution. Annu Rev Ecol Evol Syst 38:231-253

Saltré F, Saint-Amant R, Gritti ES et al (2013) Climate or migration: what limited European beech postglacial colonization? Glob Ecol Biogeogr 22:1217-1227. doi:10.1111/geb.12085

Satterthwaite WH (2010) Competition for space can drive the evolution of dormancy in a temporally invariant environment. Plant Ecol 208:167-185. doi:10.1007/s11258-009-9696-y

Schaal BA (1980) Reproductive capacity and seed size in Lupinus texensis. Am J Bot 67:703-709. doi:10. $2307 / 2442663$

Snyder RE (2006) Multiple risk reduction mechanisms: can dormancy substitute for dispersal? Ecol Lett 9:1106-1114. doi:10.1111/j.1461-0248.2006.00962.x

Soons MB, Van Der Vlugt C, Van Lith B et al (2008) Small seed size increases the potential for dispersal of wetland plants by ducks. J Ecol 96:619-627. doi:10.1111/j.1365-2745.2008.01372.x

Thiede DA, Augspurger CK (1996) Intraspecific variation in seed dispersion of Lepidium campestre (Brassicaceae). Am J Bot 83:856-866

Thomas CD, Cameron A, Green RE et al (2004) Extinction risk from climate change. Nature 427:145-148. doi:10.1038/nature02121

Traveset A (1998) Effect of seed passage through vertebrate frugivores' guts on germination: a review. Perspect Plant Ecol Evol Syst 1(2):151-190

Tsuji N, Yamamura N (1992) A simple evolutionary model of dormancy and dispersal in heterogeneous patches with special reference to phytophagous lady beetles: I. Stable environments. Res Popul Ecol 34:77-90. doi:10.1007/BF02513523 
Vander Wall SB, Longland WS (2004) Diplochory: are two seed dispersers better than one? Trends Ecol Evol 19:155-161. doi:10.1016/j.tree.2003.12.004

Venable DL (2007) Bet hedging in a guild of desert annuals. Ecology 88:1086-1090

Venable DL, Brown JS (1988) The selective interactions of dispersal, dormancy, and seed size as adaptations for reducing risk in variable environments. Am Nat 131:360-384

Venable DL, Lawlor L (1980) Delayed germination and dispersal in desert annuals-escape in space and time. Oecologia 46:272-282

Vitalis R, Rousset F, Kobayashi Y et al (2013) The joint evolution of dispersal and dormancy in a metapopulation with local extinctions and kin competition. Evolution 67:1676-1691. doi:10.1111/evo. 12069

Walther GR, Post E, Convey P et al (2002) Ecological responses to recent climate change. Nature 416:389-395

Waser NM, Price MV, Shaw RG (2000) Outbreeding depression varies among cohorts of Ipomopsis aggregata planted in nature. Evolution 54:485-491. doi:10.1111/j.0014-3820.2000.tb00051.x

Wender NJ, Polisetty CR, Donohue K (2005) Density-dependent processes influencing the evolutionary dynamics of dispersal: a functional analysis of seed dispersal in Arabidopsis thaliana (Brassicaceae). Am J Bot 92:960-971

Wiener P, Tuljapurkar S (1994) Migration in variable environments: exploring life-history evolution using structured population models. J Theor Biol 166:75-90. doi:10.1006/jtbi.1994.1006

Wilczek AM, Roe JL, Knapp MC et al (2009) Effects of genetic perturbation on seasonal life history plasticity. Science 323:930-934. doi:10.1126/science.1165826

Wilczek AM, Cooper MD, Korves TM, Schmitt J (2014) Lagging adaptation to warming climate in Arabidopsis thaliana. Proc Natl Acad Sci 111:7906-7913. doi:10.1073/pnas.1406314111

Willis KJ, MacDonald GM (2011) Long-term ecological records and their relevance to climate change predictions for a warmer world. Annu Rev Ecol Evol Syst 42:267-287. doi:10.1146/annurev-ecolsys102209-144704

Willis CG, Ruhfel B, Primack RB et al (2008) Phylogenetic patterns of species loss in Thoreau's woods are driven by climate change. Proc Natl Acad Sci USA 105:17029-17033. doi:10.1073/pnas.0806446105

Willis KJ, Bailey RM, Bhagwat SA, Birks HJB (2010a) Biodiversity baselines, thresholds and resilience: testing predictions and assumptions using palaeoecological data. Trends Ecol Evol 25:583-591. doi:10. $1016 / j$.tree.2010.07.006

Willis KJ, Bennett KD, Bhagwat SA, Birks HJB (2010b) $4{ }^{\circ} \mathrm{C}$ and beyond: what did this mean for biodiversity in the past? Syst Biodivers 8:3-9. doi:10.1080/14772000903495833

Willis KJ, Bennett KD, Burrough SL et al (2013) Determining the response of African biota to climate change: using the past to model the future. Philos Trans R Soc Lond B Biol Sci 368:20120491. doi:10. 1098/rstb.2012.0491

Willis CG, Hall JC, de Casas RR et al (2014) Diversification and the evolution of dispersal ability in the tribe Brassiceae (Brassicaceae). Ann Bot. doi:10.1093/aob/mcu196 